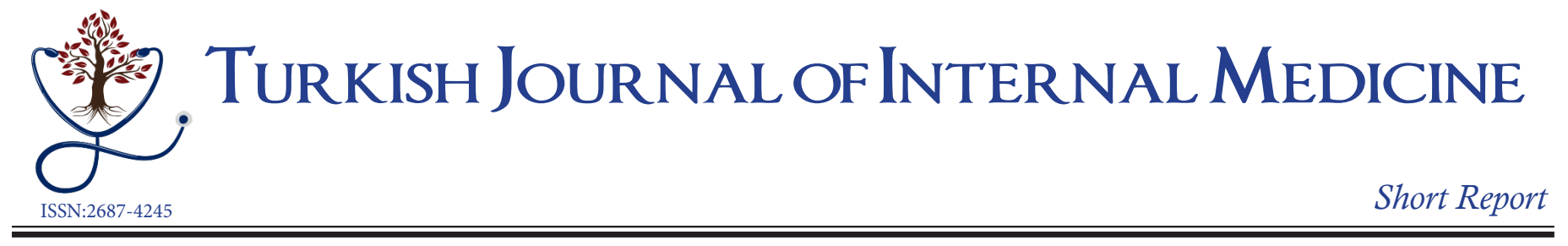

\title{
Prognostic Biomarkers in Lung Cancer Patients in terms of Long-term Survival
}

\author{
Belkıs Nihan COŞKUN¹, Oğuzhan Sıtkı DİZDAR², Şeniz KORKMAZ, Engin ULUKAYA4, Türkkan \\ EVRENSEL ${ }^{5}$

\footnotetext{
${ }^{1}$ Bursa Uludağ University Faculty of Medicine, Department of Internal Diseases, Division of Rheumatology, Bursa, Turkey

${ }^{2}$ Kayseri City Training and Research Hospital, Internal Medicine Clinic, Kayseri, Turkey

${ }^{3}$ Karacabey State Hospital Biochemistry Department, Bursa, Turkey

${ }^{4}$ Istinye University, Department of Biochemistry, Istanbul, Turkey

${ }^{5}$ Bursa Uludağ University Faculty of Medicine, Department of Internal Diseases, Division of Oncology, Bursa, Turkey
}

Turk J Int Med 2021;3(Supplement 1):S20-S22

DOI: $10.46310 /$ tjim.8875437

Keywords: Lung cancer, M30 and M65 antigen, neoadjuvant therapy, long-term prognosis

Lung cancer is the leading cause of death associated with cancer worldwide. The most common lung cancer is non-small-cell lung cancer (NSCLC). Drugs used in the treatment of NSCLC, such as paclitaxel, induce microtubule stabilization in cancer cells, resulting in mitotic arrest in a subset of cancer cells accompanied by apoptotic cell death. ${ }^{1}$ Circulating fragments of cytokeratin 18 are $\mathrm{M} 30$ released during apoptosis and M65 released during necrosis and are known to be important markers for evaluating the chemotherapy response, especially in epithelial cancers. ${ }^{2}$ In this study, we aimed to investigate the predictive effect of serum M30 and M65 antigens on long-term prognosis and in patients with advanced stage lung cancer before and after the first dose of chemotherapy.

Forty-eight patients with advanced stage lung cancer were included in the study. Demographic data and histopathological characteristics of the patients were recorded. Serum levels of M30 and M65 were studied in 48 patients before chemotherapy, and in 43 patients both before and 48 hours after chemotherapy. Long-term survival was evaluated. The median value of M30 and M65 of all patients was selected as the cut-off point for the distinction of M30 and M65 -high patients from M30 and M65 -low patients. The effect of high or low M30, M65 levels and M30/M65 ratio on long-term survival was investigated. Statistical analyses were performed using SPSS 22.0 (SPSS Inc., Chicago, IL, USA) software. The mean serum M30 and M65 values which were calculated before and after chemotherapy were compared using the paired sample t-test. According to the KaplanMeier process, survival analysis and curves have

Received:February 08,2021;Accepted:March 4, 2021; Published Online:March 6, 2021

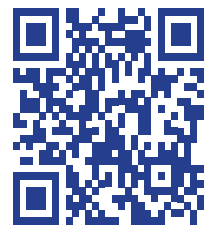


been developed and compared by the log-rank test.

The mean age of the patients at the time of diagnosis was $57.52 \pm 9.38$ years. Forty-six of the 48 patients were men. $47.9 \%$ of the patients were stage IIIA, $39.6 \%$ stage IIIB and $12.5 \%$ stage IV. The most dominant histopathological type was squamous cell carcinoma, accounting for $87.5 \%$ of the patients. The remaining $8.3 \%$ patients had adenocarcinoma and $4.2 \%$ patients had large cell carcinoma.

While M30 value before chemotherapy was $163.23 \pm 112.30 \mathrm{U} / \mathrm{L}$; It was measured as $249.74 \pm 266.67 \mathrm{U} / \mathrm{L} 48$ hours after chemotherapy $(\mathrm{p}<0.001)$. While M65 value before chemotherapy was $415.97 \pm 214.63 \mathrm{U} / \mathrm{L}$; It was $656.65 \pm 394.15$ U/L 48 hours after chemotherapy $(\mathrm{p}<0.001)$. Median survival time was calculated as 17 (2-142) months. It was found that the long-term survival of the group with a low M30/M65 ratio before chemotherapy was statistically significantly longer than the group with a high M30/M65 ratio (Figure 1). It was observed that the M30/ M65 ratio, or M30 or M65 alone, measured 48 hours after chemotherapy had no predictive value for long-term survival. Chemotherapeutic agents such as paclitaxel is the mainstay of the treatment in advanced lung cancer. It is crucial to identify best candidates for the treatment. ${ }^{1}$

M30 and M65 are two distinctive fragments of cytokeratin 18. While M30 is released during apoptosis; M65 is released during necrosis.2 M30 was used for determination of apoptosis in many cancers including head and neck tumors, pancreatic cancer, colorectal carcinoma, melanoma, nasopharyngeal carcinoma, breast cancer, etc. ${ }^{3.9}$ The role of M30 and M65 also investigated in lung cancer. ${ }^{10}$

In our previous work, we demonstrated increased levels of both M30 and M65 in this patient cohort when compared to healthy individuals. ${ }^{11}$ In this study we aimed to evaluate the predictive effect of these markers on long term prognosis. We performed Kaplan-Meier curves depending on lower and higher levels of M30, M65 and the ratio of M30/M65 before and after the chemotherapy. Only higher ratio of M30/M65 was found to be statistically significant for longer overall survival. We speculate the higher levels of baseline apoptotic activity is important for treatment response of taxane based chemotherapy. In their study, Chu et al. ${ }^{12}$ compared the ratio of M30/M65 in advanced lung cancer who had administered paclitaxel therapy. Unlike our results, they found that lower rates of M30/M65 after chemotherapy was a significant predictor of long-term survival. They explained this link with reduced activation of necrotic cell death by chemotherapy with paclitaxel has a better effect on treatment with NSCLC. Also, according to their hypothesis this may be due to activation

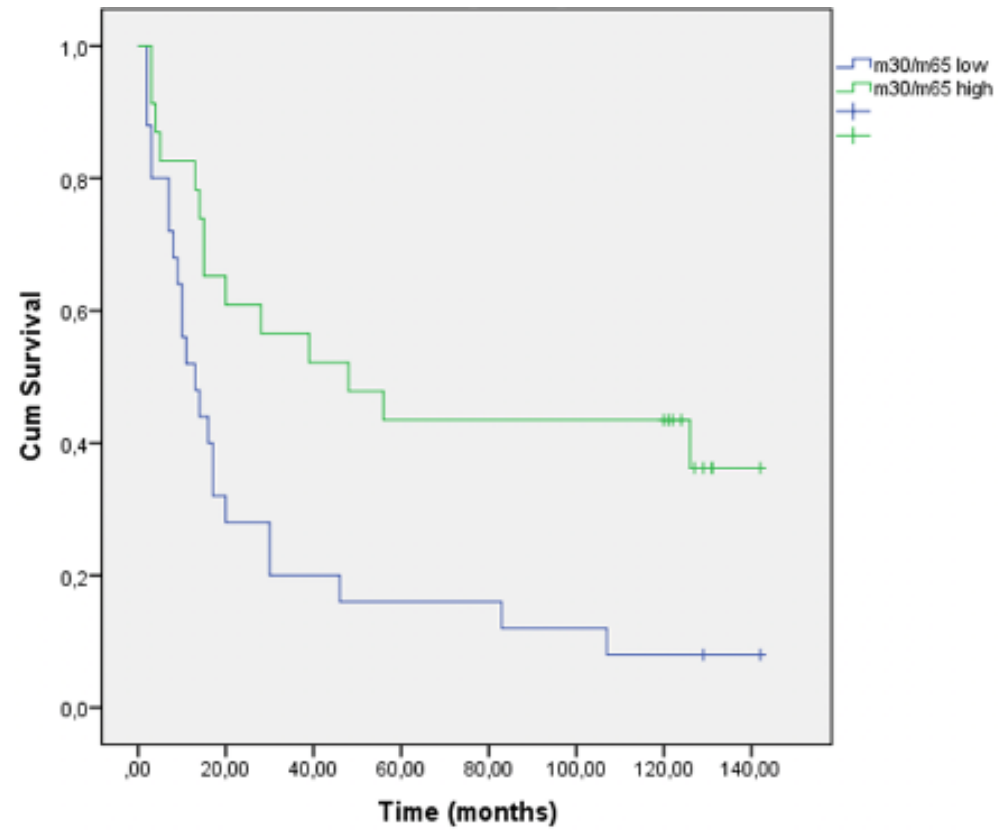

Figure 1. Comparison of survival rates regarding M30/M65 ratio before chemotherapy. 
in NSCLC cells of the classic caspase-mediated apoptosis mechanism. ${ }^{12}$

In conclusion, the $\mathrm{M} 30 / \mathrm{M} 65$ ratio before chemotherapy may be a prognostic factor for long-term survival in patients with advanced lung cancer. In order to better determine the importance of these prognostic markers, multi-center studies with a higher number of patients are needed.

\section{Conflict of Interests}

Authors declare that there are none.

\section{Acknowledgment}

This study has been presented in $17^{\text {th }}$ Uludag Internal Medicine National Winter Congress, $6^{\text {th }}$ Bursa Family Medicine Association National Congress, $11^{\text {th }}$ Uludag Internal Medicine Nursing Congress, 5-7 March 2021, Bursa, Turkey.

\section{References}

1. Caramori G, Casolari P, Cavallesco GN, Giuffrè S, Adcock I, Papi A. Mechanisms involved in lung cancer development in COPD. Int J Biochem Cell Biol. 2011 Jul;43(7):1030-44. doi: 10.1016/j.biocel.2010.08.022.

2. Ueno T, Toi M, Linder S. Detection of epithelial cell death in the body by cytokeratin 18 measurement. Biomed Pharmacother. 2005 Oct;59 Suppl 2:S359-62. doi: 10.1016/s0753-3322(05)80078-2.

3. Ozturk B, Coskun U, Sancak B, Yaman E, Buyukberber S, Benekli M. Elevated serum levels of M30 and M65 in patients with locally advanced head and neck tumors. Int Immunopharmacol. 2009 May;9(5):645-8. doi: 10.1016/j.intimp.2009.02.004.

4. Bilici A, Ustaalioglu BB, Ercan S, Orcun A, Seker M, Salepci T, Gumus M. Is there any impact of plasma M30 and M65 levels on progression-free survival of patients with advanced gastric cancer? Cancer Chemother Pharmacol. 2011 Aug;68(2):309-16. doi: 10.1007/s00280-010-1480-0.

5. Yaman E, Coskun U, Sancak B, Buyukberber S, Ozturk B, Benekli M. Serum M30 levels are associated with survival in advanced gastric carcinoma patients. Int Immunopharmacol. $2010 \mathrm{Jul} ; 10(7): 719-22$. doi: 10.1016/j.intimp.2010.03.013.

6. Ausch C, Buxhofer-Ausch V, Olszewski U, Schiessel R, Ogris E, Hinterberger W, Hamilton G. Circulating cytokeratin 18 fragment $\mathrm{m} 65$-a potential marker of malignancy in colorectal cancer patients. J Gastrointest Surg. 2009 Nov;13(11):2020-6. doi: 10.1007/s11605009-0992-6.

7. Sen F, YildizI, Odabas H, Tambas M, KilicL, Karadeniz A, Altun M, Ekenel M, Serilmez M, Duranyildiz D, Bavbek S, Basaran M. Diagnostic value of serum M30 and M65 in patients with nasopharyngeal carcinoma. Tumour Biol. 2015 Feb;36(2):1039-44. doi: 10.1007/ s13277-014-2708-0.

8. Tas F, Karabulut S, Serilmez M, Yildiz I, Sen F, Ciftci $\mathrm{R}$, Duranyildiz D. Clinical significance of serum M30 and M65 levels in melanoma. Melanoma Res. 2013 Oct;23(5):390-5. doi: 10.1097/CMR.0b013e328363e4ab.

9. Tas F, Karabulut S, Bilgin E, Sen F, Yildiz I, Tastekin D, Ciftci R, Duranyildiz D. Clinical significance of serum M30 and M65 levels in metastatic pancreatic adenocarcinoma. Tumour Biol. 2013 Dec;34(6):352936. doi: 10.1007/s13277-013-0931-8.

10. Ulukaya E, Yilmaztepe A, Akgoz S, Linder S, Karadag $\mathrm{M}$. The levels of caspase-cleaved cytokeratin 18 are elevated in serum from patients with lung cancer and helpful to predict the survival. Lung Cancer. 2007 Jun;56(3):399-404. doi: 10.1016/j.lungcan.2007.01.015.

11. Coskun BN, Dizdar OS, Korkmaz S, Ulukaya E, Evrensel T. The roles of M30 and M65 in the assessment 